\title{
Quantum gravity effect in torsion driven inflation and $\mathrm{CP}$ violation
}

\author{
Sayantan Choudhury, ${ }^{a, 1}$ Barun Kumar Pal, ${ }^{b, c}$ Banasri Basu $^{d}$ and \\ Pratul Bandyopadhyay ${ }^{d}$ \\ ${ }^{a}$ Department of Theoretical Physics, Tata Institute of Fundamental Research, \\ Colaba, 1, Homi Bhabha Road, Mumbai 400005, India \\ ${ }^{b}$ Inter-University Centre for Astronomy and Astrophysics, \\ Ganeshkhind, Pune 41100\%, India \\ ${ }^{c}$ Netaji Nagar College for Women, \\ Regent Estate, Kolkata 700092, India \\ ${ }^{d}$ Physics and Applied Mathematics Unit, Indian Statistical Institute, \\ 203 B.T. Road, Kolkata 700 108, India \\ E-mail: sayantan@theory.tifr.res.in, barunp1985@rediffmail.com, \\ sribbasu@gmail.com, b_pratul@yahoo.co.in
}

ABSTRACT: We have derived an effective potential for inflationary scenario from torsion and quantum gravity correction in terms of the scalar field hidden in torsion. A strict bound on the $\mathrm{CP}$ violating $\theta$ parameter, $\mathcal{O}\left(10^{-10}\right)<\theta<\mathcal{O}\left(10^{-9}\right)$ has been obtained, using Planck+WMAP9 best fit cosmological parameters.

KEYwords: Models of Quantum Gravity, Cosmology of Theories beyond the SM, Effective field theories, Chern-Simons Theories

ARXiv EPRINT: 1409.6036

\footnotetext{
${ }^{1}$ Presently working as a Visiting (Post-Doctoral) fellow at DTP, TIFR, Mumbai, India.
} 


\section{Contents}

1 Introduction 1

2 Torsion induced potential 1

3 Inflationary modeling with the CP violating term 3

4 The effective potential 5

5 Estimate on the CP violation term $\quad 6$

\begin{tabular}{llr}
6 & Discussion & 8 \\
\hline
\end{tabular}

\section{Introduction}

The paradigm of cosmic inflation complements the big-bang theory and when combined together it is the best theory compatible with the latest observations. Inflation is generally believed to be driven by a scalar field known as inflaton. Gasperini [1] has pointed out that the inflationary scenario can be well explained through torsion. ${ }^{1}$ Later, Poplawski [3] have argued that torsion can be treated as an alternative source of inflation. In this context, it is also to be noted that when torsion is considered to be generated from spin-spin interaction a hidden scalar field can be associated with torsion [4]. It is interesting to know whether the associated scalar field in torsion plays the role of inflaton in the inflationary regime such that "the scalar field driven inflation" as well as the "torsion driven inflation" appear to be equivalent statements. The motivation of the present paper is to show that if in the Einstein-Cartan-Kibble-Sciama (ECKS) theory of gravity, the quantum gravity effect in the early universe is taken into account, we can formulate an effective potential for inflation in terms of the scalar field hidden in the torsion. Besides, the formulation gives rise to a $\mathrm{CP}$ violating term. The estimate of the bound on $\mathrm{CP}$ violation in the early Universe using Planck+WMAP9 best fit cosmological parameters $[5,6]$ has also been obtained.

\section{Torsion induced potential}

To study torsion in terms of the spin-spin interaction we take resort to a spin-current duality relation so that the action for torsion can be developed through a dual currentcurrent interaction. We consider a four vector $n_{\mu}$ in terms of the spinorial variables as

$$
n_{\mu}=\left(\frac{1}{\sqrt{2}}\right)\left(\begin{array}{ll}
\psi_{1}^{*} & \psi_{2}^{*}
\end{array}\right) \sigma_{\mu}\left(\begin{array}{l}
\psi_{1} \\
\psi_{2}
\end{array}\right)
$$

\footnotetext{
${ }^{1}$ Also it is important to note that, in ref. [2], the authors have explicitly studied the late-time cosmic acceleration from torision and the emergent scalar degree of freedom arose from the BCS condensation of the fermions.
} 
where

$$
\psi_{1}=(\cos \theta / 2) e^{i \phi / 2}, \quad \psi_{2}=(\sin \theta / 2) e^{-i \phi / 2},
$$

with $\sigma_{0}=I$, where $I$ is the identity matrix and $\vec{\sigma}$ is the vector of Pauli matrices. Using this one can construct an $\mathrm{SU}(2)$ group element

$$
g=n_{0} I+i \overrightarrow{\mathbf{n}} \cdot \vec{\sigma}
$$

in terms of which we can construct the topological current as [7]:

$$
J_{\mu}=\left(\frac{1}{24 \pi^{2}}\right) \epsilon_{\mu \nu \lambda \sigma} \operatorname{Tr}\left[\left(g^{-1} \partial^{\nu} g\right)\left(g^{-1} \partial^{\lambda} g\right)\left(g^{-1} \partial^{\sigma} g\right)\right]
$$

where $\epsilon_{\mu \nu \lambda \sigma}$ is the rank-4 Levi-Civita tensor. Now by demanding that in 4-dimensional Euclidean space the field strength $F_{\mu \nu}$ of a gauge potential vanishes on the boundary $S^{3}$ of a certain volume $\mathrm{Vol}_{4}$ inside of which $F_{\mu \nu} \neq 0$, we can write the gauge potential as $A_{\mu}=g^{-1} \partial_{\mu} g \in \mathrm{SU}(2)$. Then from eq. (2.4) the Kac-Moody like current $J_{\mu}$ can be recast in terms of the Chern-Simons secondary characteristic class as [8]:

$$
J_{\mu}=\left(\frac{1}{16 \pi^{2}}\right) \epsilon^{\mu \nu \lambda \sigma} \operatorname{Tr}\left(A_{\nu} F_{\lambda \sigma}+\frac{2}{3} A_{\nu} A_{\lambda} A_{\sigma}\right)
$$

This gives rise to a topological invariant:

$$
Q_{P}=\left(\frac{1}{16 \pi^{2}}\right) \int d^{4} x \partial_{\mu} J^{\mu}
$$

which is known as the Pontryagin index. We can construct the Lagrangian from the divergence of the current $J_{\mu}$ and write

$$
\mathcal{L}=-\frac{1}{4} \operatorname{Tr}\left(\epsilon_{\mu \nu \lambda \sigma} F^{\mu \nu} F^{\lambda \sigma}\right)
$$

which leads to the construction of the current [9]

$$
\mathbf{j}^{\mu}=\epsilon^{\mu \nu \lambda \sigma} \mathbf{a}_{\nu} \otimes \mathbf{f}_{\lambda \sigma}=\epsilon^{\mu \nu \lambda \sigma} \partial_{\nu} \mathbf{f}_{\lambda \sigma}
$$

with $A_{\mu}=\mathbf{a}_{\mu} \cdot \sigma$ and

$$
F_{\mu \nu}=\partial_{[\mu} A_{\nu]}+\left[A_{\mu}, A_{\nu}\right]=\mathbf{f}_{\mu \nu} \cdot \sigma
$$

It can be shown that the axial vector current

$$
J_{\mu}^{5}=\bar{\psi} \gamma_{\mu} \gamma_{5} \psi
$$

is related to the second component of the current $\mathbf{j}_{\mu}$ through the relation

$$
\partial^{\mu} j_{\mu}^{(2)}=-\frac{1}{2} \partial^{\mu} J_{\mu}^{5} \neq 0 .
$$

The consistency of the current conservation equations implies that [10]:

$$
j_{\mu}^{(1)}=-\frac{1}{2} j_{\mu}^{(2)}, j_{\mu}^{(3)}=+\frac{1}{2} j_{\mu}^{(2)}
$$


Consequently, the current-current interaction can be expressed in terms of $j_{\mu}^{(2)}$ only which effectively displays the spin-spin interaction. Now we can write the action for torsion as [11]

$$
S_{T}=\frac{M_{p}^{2}}{2} \int J_{\mu}^{2} J_{\mu}^{2} d^{4} x
$$

where $M_{p}$ being the reduced Planck mass, given by $M_{p} \approx 2.43 \times 10^{18} \mathrm{GeV}$. It is now observed that there is a hidden scalar field $\phi$ in torsion which follows from the relation

$$
j^{\mu(2)}=\epsilon^{\mu \nu \lambda \sigma} \partial_{\nu} f_{\lambda \sigma}^{(2)}=\epsilon^{\mu \nu \lambda \sigma} \epsilon_{\nu \lambda \sigma} \phi(x)
$$

where $\epsilon^{\nu \lambda \sigma}$ is the rank-3 Levi-Civita tensor. The action now turns out to be:

$$
S_{T}=\mathcal{A} \int d^{4} x j_{\mu}^{(2)} j^{\mu(2)}=\int d^{4} x \sqrt{-g_{(4)}} \frac{m^{2}}{2} \phi^{2}
$$

eq. (2.15) suggests that the potential associated with torsion can be written as:

$$
V_{T}(\phi)=-\frac{m^{2}}{2} \phi^{2}
$$

The negative sign of the coupling constant $m^{2}$ actually corresponds to the self interaction, when orientation of all the spin degrees of freedom are along the same direction.

\section{Inflationary modeling with the $\mathrm{CP}$ violating term}

Now we analyse the contribution from quantum gravity. To this end we utilize the model of Capovilla, Jacobson and Drell (CJD) [12, 13], where the action is given by [12, 13]:

$$
S=\frac{1}{8} \int \eta\left(\Omega_{i j} \Omega_{i j}+a \Omega_{i i} \Omega_{j j}\right)
$$

where

$$
\Omega_{i j}=\epsilon^{\alpha \beta \gamma \delta} F_{\alpha \beta i} F_{\gamma \delta j}
$$

with $\alpha, \beta, \gamma, \delta$ as space time indices, $i, j$ the $\mathrm{SU}(2)$ group indices and $\eta$ is a scalar density. In ref. $[12,13]$ it has been shown that in $3+1$ decomposition this action yields Ashteker action directly provided we have $a=-\frac{1}{2}$ and the determinant of the magnetic field $B^{i}{ }_{a}$ is non zero and as such the equivalence to the Einstein's theory is established. The equivalence to the Einstein's theory can also be shown when the space time metric is found to be given by

$$
\sqrt{-g_{(4)}} g^{\alpha \beta}=-\left(\frac{2 i}{3 \eta}\right) \epsilon_{i j k} \epsilon^{\alpha \gamma \delta \rho} \epsilon^{\beta \mu \nu \sigma} F_{\gamma \delta i} F_{\rho \sigma j} F_{\mu \nu k}
$$

The constraint that is obtained when the CJD action is varied with respect to the Lagrangian multiplier $\eta$ is actually the Hamiltonian constraint

$$
\Psi=\Omega_{i j} \Omega_{i j}-\frac{1}{2} \Omega_{i i} \Omega_{j j}=i\left(2 \eta^{2} \operatorname{det} B\right)^{-1} H
$$


This implies that $\Psi \approx 0$ and $H \approx 0$ are equivalent statements provided $\operatorname{det} B \neq 0$. The canonical transformation of $\mathrm{SU}(2)$ gauge potential $\left(A_{a i}\right)$ and the corresponding non-abelian fields $\left(E_{i}^{a}, B_{i}^{a}\right)$ :

$$
\begin{aligned}
A_{a i} & \rightarrow A_{a i}, \\
E_{i}^{a} & \rightarrow E_{i}^{a}-\theta B_{i}^{a}
\end{aligned}
$$

gives rise to a CP-violating $\theta$ term in the CJD Lagrangian so that for $a=-1 / 2$ the action now reads $[4,12-15]$ :

$$
S_{C}=\frac{1}{8} \int\left[\theta \Omega_{i i}+\eta\left(\Omega_{i j} \Omega_{i j}-\frac{1}{2} \Omega_{i i} \Omega_{j j}\right)\right] .
$$

In the first term the parameter $\theta$ essentially corresponds to the measure of $\mathrm{CP}$ violation which contributes to torsion and the rest is curvature contribution. Consequently eq. (3.7) can be recast as:

$$
\begin{aligned}
S_{C}= & -\frac{\theta}{4} Q_{P}+\eta \int d^{4} x \epsilon^{\alpha \beta \gamma \delta} \epsilon^{\lambda \rho \sigma \mu} \epsilon_{\nu \alpha \beta} \epsilon_{\nu^{\prime} \lambda \rho} \epsilon_{\xi \nu \delta} \epsilon_{\xi^{\prime} \sigma \mu} \int d x^{\nu} \phi \int d x^{\nu^{\prime}} \phi \int d x^{\xi} \phi \int d x^{\xi^{\prime}} \phi \\
& -\frac{\eta}{2} \int d^{4} x \epsilon^{\mu \nu \lambda \sigma} \epsilon^{\alpha \beta \gamma \delta} \epsilon_{\nu \lambda \sigma} \epsilon_{\beta \gamma \delta}\left(\partial_{\mu} \phi\right)\left(\partial_{\alpha} \phi\right)
\end{aligned}
$$

where $\int d x^{\nu} \phi=\phi\left[x^{\nu}\right]$, and the symbol $[\cdots]$ signifies the boundary value of the coordinates in the affine parameter space. Now from eq. (3.8) we get: ${ }^{2}$

$$
S_{C}=-\frac{\theta}{4} Q_{P}+\int d^{4} x \sqrt{-g_{(4)}}\left[\frac{g^{\mu \alpha}}{2}\left(\partial_{\mu} \phi\right)\left(\partial_{\alpha} \phi\right)-\frac{\lambda}{4} \phi^{4}\right] .
$$

It may be mentioned here that the first term on the right hand side incorporates the Pontryagin index given by eq.(2.6) which is a topological term arising from a total divergence. This does not contribute classically but has the effect in the quantum mechanical formulation.

From eq. (2.15) and eq. (3.9), we note that the action for torsion (curvature) when expressed in terms of the $\phi$ field involves the term $\phi^{2}\left(\phi^{4}\right)$. This indicates that the anisotropies associated with the torsion are much suppressed in comparison to the contribution from curvature for large values of $\phi$. It is noted that the the expression of curvature in terms of the scalar field arises when we use CJD Lagrangian. In this sense the scalar field does not arise from gravitation as such, but it originates from the torsional degrees of freedom associated with the spin density.

Noting that the asymptotic constancy of torsion compensates the bare cosmological constant [16] we can define a small but non-vanishing cosmological constant in terms of the Pontryagin index as

$$
M_{p}^{2} \Lambda_{\mathrm{eff}}=\frac{\theta}{8 \mathrm{Vol}_{4}} Q_{P}
$$

\footnotetext{
${ }^{2}$ Here we use the following spin-particle duality relations:

$$
\begin{aligned}
\eta \epsilon^{\mu \nu \lambda \sigma} \epsilon^{\alpha \beta \gamma \delta} \epsilon_{\nu \lambda \sigma} \epsilon_{\beta \gamma \delta} & =-\sqrt{-g_{(4)}} g^{\mu \alpha} \\
\eta \epsilon^{\alpha \beta \gamma \delta} \epsilon^{\lambda \rho \sigma \mu} \epsilon_{\nu \alpha \beta} \epsilon_{\nu^{\prime} \lambda \rho} \epsilon_{\xi \nu \delta} \epsilon_{\xi^{\prime} \sigma \mu}\left[x^{\nu} x^{\nu^{\prime}} x^{\xi} x^{\xi^{\prime}}\right] & =-\frac{\lambda}{4} .
\end{aligned}
$$
}


where $M_{p}$ coresponds to the Planck mass. We can define the vacuum energy $V_{0}$ through the relation

$$
V_{0}=3 H_{i n f}^{2} \Lambda_{U V}^{2}=\Lambda_{\text {eff }} \Lambda_{U V}^{2}
$$

Here $\Lambda_{U V}$ signifies the UV cut-off scale of the proposed EFT theory. ${ }^{3}$ Below $\Lambda_{U V}$ the effect of all quantum corrections are highly suppressed and the heavy fields from the hidden sector gets their VEV. Such VEV is one of the possible sources of vacuum energy correction in the spin-current dominated EFT picture which uplifts the scale of inflationary potential and the contributions of the VEV become significant upto a scale $\Lambda_{C} \leq \Lambda_{U V}$. But at very low scale, $\Lambda_{\text {low }} \ll \Lambda_{C}$, one can tune the vacuum energy correction, $V_{0} \approx 0$ for which the contributions of the VEV can be neglected $[17,18]$. Such possibility is only significant when the contribution of the primordial gravity waves become negligibly small (see eq. (5.1)). Thus the expression for the potential from CJD Lagrangian incorporating the CP violating $\theta$ term yields:

$$
V_{C}(\phi)=V_{0}+\frac{\lambda}{4} \phi^{4}
$$

\section{The effective potential}

Now in the background of a space-time manifold having Riemannian structure the contribution to the conserved current can be expressed as:

$$
J^{\mu g}=\frac{1}{2} \epsilon^{\mu \nu \lambda \sigma} R_{\nu \lambda \sigma \delta} v^{\delta},
$$

where $v^{\delta}$ is an arbitrary vector and Riemann curvature tensor can be expressed as:

$$
R_{\nu \lambda \sigma \delta}=\partial_{[\lambda} \omega_{\nu] \sigma \delta}+\omega_{\nu \sigma}^{\eta} \omega_{\lambda \eta \delta}-\omega_{\lambda \sigma}^{\xi} \omega_{\nu \xi \delta}-e_{\sigma \nu} e_{\delta \lambda}
$$

As a result the gravitational part of the action can be written in terms of gravitational current-current interaction in the Riemann space as:

$$
S_{g}=-\frac{\Lambda_{U V}^{2}}{2} \int d^{4} x J_{\mu}^{g} J^{\mu g}=\frac{\Lambda_{U V}^{2}}{2} \int d^{4} x \sqrt{-g_{4}} R
$$

Now clubbing the contributions from eqs. (2.15), (3.9), (4.3) the total action for the present field theoretic setup, taking into account quantum gravity correction, can finally be written as:

$$
S=\int d^{4} x \sqrt{-g_{4}}\left[\frac{\Lambda_{U V}^{2}}{2} R+\frac{g^{\mu \alpha}}{2}\left(\partial_{\mu} \phi\right)\left(\partial_{\alpha} \phi\right)-V(\phi)\right]
$$

\footnotetext{
${ }^{3}$ Above the scale $\Lambda_{U V}$ it is necessarily required to introduce the higher order quantum corrections to the usual classical theory of gravity represented via Einstein-Hilbert term, as the role of these corrections are significant in trans-Planckian scale to make the theory UV complete [19, 20]. However such quantum corrections are extremely hard to compute as it completely belongs to the hidden sector of the theory dominated by heavy fields [21, 22]. In the trans-Planckian regime the classical gravity sector is corrected by incorporating the effect of higher derivative interactions appearing through the modifications to GR which plays significant role in this context [23-28]. On the other hand in trans-Planckian regime quantum corrections of matter fields and their interaction between various constituents modify the picture which are appearing through perturbative loop corrections [29-32].
} 
such that the total effective potential is given by:

$$
V(\phi)=V_{T}(\phi)+V_{C}(\phi)=V_{0}-\frac{m^{2}}{2} \phi^{2}+\frac{\lambda}{4} \phi^{4} .
$$

\section{Estimate on the $\mathrm{CP}$ violation term}

The effective potential is dominated by the vacuum energy correction term which determines the scale of inflation. To obtain the scale of inflation at $k_{*} \approx k_{c m b}$, we express $V_{0}$ in terms of inflationary observables as:

$$
V_{*}^{1 / 4} \approx V_{0}^{1 / 4}=7.389 \times 10^{-3} \Lambda_{U V} \times\left(\frac{r}{0.1}\right)^{1 / 4} .
$$

where $r$ is the tensor-to-scalar ratio defined as: $r=A_{T} / A_{S}$ with $\left(A_{T}, A_{S}\right)$ being the amplitudes of the power spectra for scalar $(S)$ and tensor $(T)$ modes at $k=a H \approx k_{*}$. The effective cosmological constant or equivalently the $\mathrm{CP}$ violating parameter $\theta$ can then be constrained as:

$$
\Lambda_{\text {eff }}=\frac{\theta}{8 \mathrm{Vol}_{4}} Q_{P}=2.98 \times 10^{-9} \Lambda_{U V}^{2} \times\left(\frac{r}{0.1}\right) .
$$

In order to compare the theoretical predictions with the latest observations we use a numerical code CLASS [33]. In this code we can directly input the shape of the potential along with the model parameters. Then for a given cosmological background the code provides the estimates for different CMB observables. In the code we set the momentum pivot at $k_{*}=0.05 \mathrm{Mpc}^{-1}$ and used the Planck + WMAP9 best fit values:

$$
h=0.670, \quad \Omega_{b}=0.049, \quad \Omega_{c}=0.268, \quad \Omega_{\Lambda}=0.682
$$

for background cosmological parameters. In this work we scan the parameter space within the following window:

$$
\begin{aligned}
2.501 \times 10^{-9} \Lambda_{U V}^{4} & \leq V_{0} \leq 2.589 \times 10^{-9} \Lambda_{U V}^{4} \\
6 \times 10^{-3} \Lambda_{U V}^{-2} & \leq m^{2} / V_{0} \leq 8 \times 10^{-3} \Lambda_{U V}^{-2} \\
\lambda / V_{0} & \sim 10^{-6} \Lambda_{U V}^{-4}
\end{aligned}
$$

As a result, the CMB observables are constrained within the following range:

$$
\begin{aligned}
2.197 \times 10^{-9} & \leq A_{S} \leq 2.202 \times 10^{-9}, \\
0.957 & \leq n_{S} \leq 0.962, \\
-1.08 \times 10^{-3} & \leq \alpha_{S} \leq-0.99 \times 10^{-3}, \\
0.055 & \leq r \leq 0.057 .
\end{aligned}
$$

Within the present context the field excursion [34-43] is defined as:

$$
|\Delta \phi|=\Lambda_{U V} \int_{0}^{N_{c m b}} d N \sqrt{\frac{r(N)}{8}} \approx \sqrt{\frac{r}{8}} N_{c m b} \Lambda_{U V} .
$$




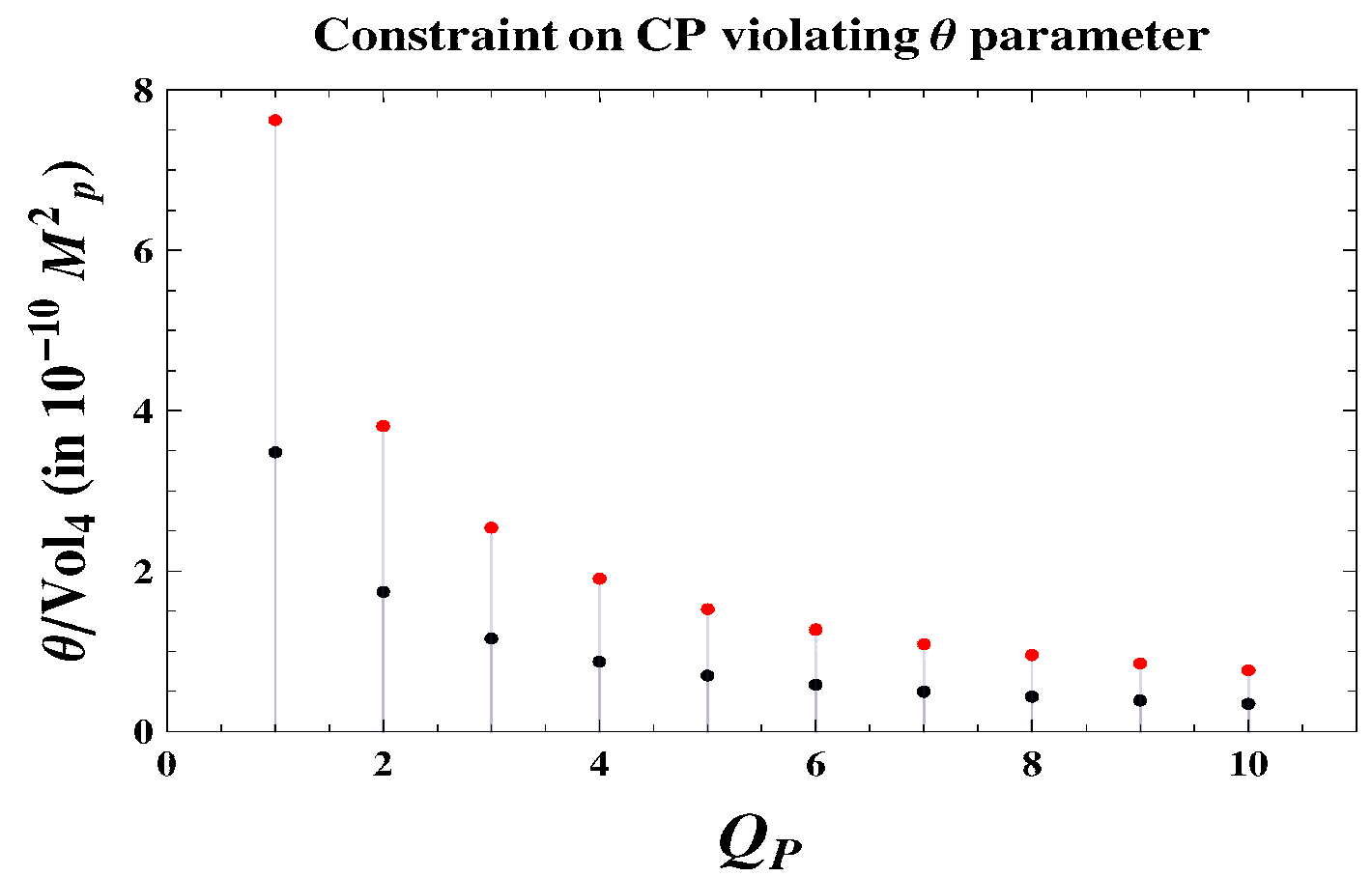

Figure 1. Constraint on $\mathrm{CP}$ violating topological $\theta$ parameter for discrete integer values of Pontryagin index $Q_{P}$ using Planck + WMAP9 best fit cosmological parameters. Here Red and black colored points correspond to the upper and lower bound of the $\theta$ parameter for a given value of $Q_{P}$. All the parallel blue colored lines are drawn for different integer values of $Q_{P}$ which connects both the Red and black colored points. This plot suggests that as the value of $Q_{P}$ increases then the interval between the upper and lower bound of the $\theta$ parameter decrease and it will converge to very small value for large $Q_{P}$. Also the numerical value corresponding to the upper bound and lower bound of the $\theta$ parameter decreases once we increase the the value of $Q_{P}$.

where $|\Delta \phi|=\left|\phi_{*}-\phi_{f}\right|$, in which $\phi_{*}$ and $\phi_{f}$ represent the field value corresponding to CMB scale and end of inflation respectively. Also $N_{c m b}$ is the number of e-foldings at CMB scale which is fixed at $N_{c m b} \approx 50-70$ to solve the horizon problem associated with inflation. Subsequently we get the following constraint on the field excursion:

$$
|\Delta \phi| \sim \mathcal{O}(4.1-5.9) \times \Lambda_{U V}
$$

which implies to make the EFT of inflation validate within the prescribed setup for which we need to constrain the UV cut-off of the EFT within the following window:

$$
\Lambda_{U V} \sim \mathcal{O}(0.16-0.24) M_{p}<M_{p}
$$

which is just below the scale of reduced Planck mass. Finally using eq. (5.2) we get the following bound on the CP violating parameter: ${ }^{4}$

$$
3.48 \times 10^{-10} M_{p}^{2} \leq \frac{\theta}{V o l_{4}} Q_{P} \leq 7.62 \times 10^{-10} M_{p}^{2} .
$$

\footnotetext{
${ }^{4}$ From experimental measurements of the neutron electric dipole moment, the experimental limit on the $\mathrm{CP}$ violating $\theta$ parameter is $\theta \leq 10^{-9}$ [44], which is consistent with our derived stringent bound on $\theta$.
} 


\section{Discussion}

Thus once we fix $Q_{P}$, this will further provide an estimate of $\theta$ according to the eq. (5.9). In figure (1) we have explicitly shown the constraint on $\theta$ from the proposed EFT picture which is obtained by using Planck + WMAP9 best fit cosmological parameters. To exemplify we have prescribed the bound on $\theta$ for different integer values of $Q_{P}$ lying within $1 \leq Q_{P} \leq 10$. From the plot it is easy to see that as the value of $Q_{P}$ increases the bound on the parameter $\theta$ converges to a very small value. This suggests that $\theta$ will converge to a constant value beyond a certain value of $Q_{P}$. It may be mentioned that the Pontryagin index can be taken to correspond to the fermion number $[45,46]$. Indeed a fermion can be realized as a scalar particle encircling a vortex line which is topologically equivalent to a magnetic flux line and thus represents a skyrmion $[45,46]$. The monopole charge $\mu=1 / 2$ corresponding to a magnetic flux line is related to the Pontryagin index through the relation $Q_{P}=$ $2 \mu$. In view of this, one may note that $Q_{P}$ represents the fermion number which is the topological index carried by a fermion. For an anti-fermion $Q_{P}$ takes the negative value. In any system the effective fermion number is given by the difference between the number of fermions and anti-fermions. Thus we can quantify the fermionic matter and hence the spin density through the total accumulated value of $Q_{P}$. As $Q_{P}$ increases we have the increase of fermions implying the increase in spin density. So from eq. (5.9) we note that for a fixed volume when $Q_{P}$ increases indicating the increase in spin density, the bound on the parameter $\theta$ converges to a small value representing the residual effect of torsion residing at the boundary. Thus the remnant of $\mathrm{CP}$ violation ${ }^{5}$ giving rise to torsion can be witnessed through the small value of $\theta$ which is operative at the boundary.

To summarize, we have derived an effective potential for inflationary scenario, taking into account the quantum gravity effect, in terms of the hidden scalar field associated with torsion along with a $\mathrm{CP}$ violating term.Using this we give an estimate of inflationary CMB observables by constraining the model parameters- vacuum energy, mass and selfcoupling from Planck + WMAP9 best fit values of the cosmological parameters. Finally, for the first time we constrain the $\mathrm{CP}$ violating topological $\theta$ parameter from the vacuum energy correction within EFT.

\section{Acknowledgments}

SC would like to thank Department of Theoretical Physics, Tata Institute of Fundamental Research, Mumbai for providing me Visiting (Post-Doctoral) Research Fellowship. SC take this opportunity to thank sincerely to Prof. Sandip P. Trivedi, Prof. Shiraz Minwalla, Prof. Soumitra SenGupta, Prof. Sudhakar Panda, Prof. Varun Sahni, Prof. Sayan Kar, Prof. Sudhakar Panda, Dr. Subhabrata Majumdar and Dr. Supratik Pal for their constant support and inspiration. SC take this opportunity to thank all the active members and the

\footnotetext{
${ }^{5}$ In the context of canonical quantization of gravity it is observed that for small but non-vanishing value of the cosmological constant an exact solution to all the constraints of quantum gravity is given by the Chern-Simons state that describes the vacuum at the Planck scale which is chiral and implies an inherent CP-violation in quantum gravity [47].
} 
regular participants of weekly student discussion meet "COSMOMEET" from Department of Theoretical Physics and Department of Astronomy and Astrophysics, Tata Institute of Fundamental Research for their strong support. Last but not the least, we would all like to acknowledge our debt to the people of India for their generous and steady support for research in natural sciences, especially for theoretical high energy physics, string theory and cosmology.

Open Access. This article is distributed under the terms of the Creative Commons Attribution License (CC-BY 4.0), which permits any use, distribution and reproduction in any medium, provided the original author(s) and source are credited.

\section{References}

[1] M. Gasperini, Spin dominated inflation in the Einstein-Cartan theory, Phys. Rev. Lett. 56 (1986) 2873 [INSPIRE].

[2] S. Alexander, T. Biswas and G. Calcagni, Cosmological Bardeen-Cooper-Schrieffer condensate as dark energy, Phys. Rev. D 81 (2010) 043511 [Erratum ibid. D 81 (2010) 069902] [arXiv: 0906 .5161] [INSPIRE].

[3] N.J. Popławski, Cosmology with torsion: an alternative to cosmic inflation, Phys. Lett. B 694 (2010) 181 [Erratum ibid. B 701 (2011) 672] [arXiv:1007.0587] [INSPIRE].

[4] L. Mullick and P. Bandyopadhyay, Gravity without metric, torsion and topological gravity, J. Math. Phys. 36 (1995) 370 [INSPIRE].

[5] Planck collaboration, P.A.R. Ade et al., Planck 2013 results. XXII. Constraints on inflation, Astron. Astrophys. 571 (2014) A22 [arXiv:1303.5082] [INSPIRE].

[6] Planck collaboration, P.A.R. Ade et al., Planck 2013 results. XVI. Cosmological parameters, Astron. Astrophys. 571 (2014) A16 [arXiv:1303.5076] [INSPIRE].

[7] A.G. Abanov and P.B. Wiegmann, Theta terms in nonlinear $\sigma$-models, Nucl. Phys. B 570 (2000) 685 [hep-th/9911025] [INSPIRE].

[8] S.-S. Chern and J. Simons, Characteristic forms and geometric invariants, Annals Math. 99 (1974) 48 [INSPIRE].

[9] M. Carmeli and S. Malin, Reformulation of general relativity as a gauge theory, Annals Phys. 103 (1977) 208 [INSPIRE].

[10] A. Roy and P. Bandyopadhyay, Topological aspects of a fermion and the chiral anomaly, J. Math. Phys. 30 (1989) 2366.

[11] A.K. Bandyopadhyay, P. Chatterjee and P. Bandyopadhyay, $\mathrm{SL}(2, C)$ gauge theory, $N=1$ supergravity and torsion, Gen. Rel. Grav. 18 (1986) 1193 [INSPIRE].

[12] R. Capovilla, T. Jacobson and J. Dell, General relativity without the metric, Phys. Rev. Lett. 63 (1989) 2325 [INSPIRE].

[13] R. Capovilla, T. Jacobson and J. Dell, A pure spin connection formulation of gravity, Class. Quant. Grav. 8 (1991) 59 [INSPIRE].

[14] I. Bengtsson and P. Peldan, Ashtekar's variables, the $\theta$ term and the cosmological constant, Phys. Lett. B 244 (1990) 261 [INSPIRE]. 
[15] I. Bengtsson and P. Peldan, Another 'cosmological' constant, Int. J. Mod. Phys. A 7 (1992) 1287 [INSPIRE].

[16] P. Baekler, E.W. Mielke, R. Hecht and F.W. Hehl, Kinky torsion in a Poincaré gauge model of gravity coupled to a massless scalar field, Nucl. Phys. B 288 (1987) 800 [InSPIRE].

[17] R. Allahverdi, K. Enqvist, J. García-Bellido and A. Mazumdar, Gauge invariant MSSM inflaton, Phys. Rev. Lett. 97 (2006) 191304 [hep-ph/0605035] [INSPIRE].

[18] S. Choudhury, A. Mazumdar and S. Pal, Low \& high scale MSSM inflation, gravitational waves and constraints from Planck, JCAP 07 (2013) 041 [arXiv: 1305.6398] [INSPIRE].

[19] V. Assassi, D. Baumann, D. Green and L. McAllister, Planck-suppressed operators, JCAP 01 (2014) 033 [arXiv:1304.5226] [INSPIRE].

[20] D. Baumann and L. McAllister, Inflation and string theory, arXiv:1404.2601 [INSPIRE].

[21] S. Choudhury, A. Mazumdar and E. Pukartas, Constraining $N=1$ supergravity inflationary framework with non-minimal Kähler operators, JHEP 04 (2014) 077 [arXiv:1402.1227] [INSPIRE].

[22] S. Choudhury, Constraining $N=1$ supergravity inflation with non-minimal Kähler operators using $\delta N$ formalism, JHEP 04 (2014) 105 [arXiv: 1402.1251] [INSPIRE].

[23] S. Choudhury and S. Sengupta, Features of warped geometry in presence of Gauss-Bonnet coupling, JHEP 02 (2013) 136 [arXiv: 1301.0918] [INSPIRE].

[24] S. Choudhury and S. SenGupta, Thermodynamics of charged Kalb Ramond AdS black hole in presence of Gauss-Bonnet coupling, arXiv:1306.0492 [INSPIRE].

[25] S. Choudhury, J. Mitra and S. SenGupta, Modulus stabilization in higher curvature dilaton gravity, JHEP 08 (2014) 004 [arXiv:1405.6826] [INSPIRE].

[26] S. Choudhury and S. SenGupta, A step toward exploring the features of gravidilaton sector in Randall-Sundrum scenario via lightest Kaluza-Klein graviton mass, Eur. Phys. J. C 74 (2014) 3159 [arXiv: 1311.0730] [INSPIRE].

[27] S. Choudhury, J. Mitra and S. SenGupta, Fermion localization and flavour hierarchy in higher curvature spacetime, arXiv:1503.07287 [INSPIRE].

[28] T. Biswas, E. Gerwick, T. Koivisto and A. Mazumdar, Towards singularity and ghost free theories of gravity, Phys. Rev. Lett. 108 (2012) 031101 [arXiv:1110.5249] [INSPIRE].

[29] V. Assassi, D. Baumann and D. Green, Symmetries and loops in inflation, JHEP 02 (2013) 151 [arXiv:1210.7792] [INSPIRE].

[30] L. Senatore and M. Zaldarriaga, On loops in inflation, JHEP 12 (2010) 008 [arXiv: 0912.2734] [INSPIRE].

[31] L. Senatore and M. Zaldarriaga, On loops in inflation II: IR effects in single clock inflation, JHEP 01 (2013) 109 [arXiv: 1203.6354] [INSPIRE].

[32] G.L. Pimentel, L. Senatore and M. Zaldarriaga, On loops in inflation III: time independence of zeta in single clock inflation, JHEP 07 (2012) 166 [arXiv:1203.6651] [INSPIRE].

[33] CLASS: the Cosmic Linear Anisotropy Solving System webpage, http://class-code.net/.

[34] D.H. Lyth, What would we learn by detecting a gravitational wave signal in the cosmic microwave background anisotropy?, Phys. Rev. Lett. 78 (1997) 1861 [hep-ph/9606387] [INSPIRE]. 
[35] S. Choudhury and A. Mazumdar, An accurate bound on tensor-to-scalar ratio and the scale of inflation, Nucl. Phys. B 882 (2014) 386 [arXiv:1306.4496] [INSPIRE].

[36] S. Choudhury and A. Mazumdar, Reconstructing inflationary potential from BICEP2 and running of tensor modes, arXiv:1403.5549 [INSPIRE].

[37] S. Choudhury and A. Mazumdar, Sub-Planckian inflation \& large tensor to scalar ratio with $r \geq 0.1$, arXiv: 1404.3398 [INSPIRE].

[38] S. Choudhury, Can effective field theory of inflation generate large tensor-to-scalar ratio within Randall-Sundrum single braneworld?, Nucl. Phys. B 894 (2015) 29 [arXiv: 1406.7618] [INSPIRE].

[39] S. Choudhury, Reconstructing inflationary paradigm within effective field theory framework, arXiv: 1508.00269 [INSPIRE].

[40] S. Choudhury and S. Banerjee, Hysteresis in the sky, arXiv:1506.02260 [INSPIRE].

[41] S. Choudhury, Constraining brane inflationary magnetic field from cosmoparticle physics after Planck, JHEP 10 (2015) 095 [arXiv: 1504.08206] [INSPIRE].

[42] D. Chialva and A. Mazumdar, Cosmological implications of quantum corrections and higher-derivative extension, Mod. Phys. Lett. A 30 (2015) 1540008 [arXiv:1405.0513] [INSPIRE].

[43] D. Baumann and D. Green, A field range bound for general single-field inflation, JCAP 05 (2012) 017 [arXiv:1111.3040] [INSPIRE].

[44] V. Parameswaran Nair, Quantum field theory: a modern perspective, Springer, New York U.S.A. (2005) [INSPIRE].

[45] D. Banerjee and P. Bandyopadhyay, Topological aspects of a fermion, chiral anomaly and Berry phase, J. Math. Phys. 33 (1992) 990 [inSPIRE].

[46] P. Bandyopadhyay, The geometric phase and the spin-statistics relation, Proc. Roy. Soc. London A 466 (2010) 2917.

[47] H. Kodama, Holomorphic wave function of the universe, Phys. Rev. D 42 (1990) 2548 [INSPIRE]. 